Le document suivant est la version validée (après corrections suite aux remarques des relecteurs et de la rédaction de la revue) de l'article publié dans la Revue internationale PME.

Reproduction permise par la revue et les Éditions EMS.

Pour citer l'article :

Aka, Kadia Georges, François Labelle et Michel Trépanier. 2018. « Une approche comportementale des rôles du dirigeant de PME dans le développement des innovations durables. » Revue internationale PME 31 (2). http://132.209.12.10/ojs/index.php/ripme/article/view/1063 


\title{
Une approche comportementale des rôles du dirigeant de PME dans le développement des innovations durables
}

\author{
A behavioral approach of the SME manager's roles \\ in the development of sustainability-oriented innovations
}

\author{
Una aproximación comportamental de los papeles del \\ dirigente de PYME en el desarrollo de las innovaciones \\ sostenibles
}

Kadia Georges AKA, DBA

Chercheur postdoctoral à l'Institut national de la recherche scientifique (INRS), chargé de cours à l'École de Gestion de l'Université du Québec à Trois-Rivières (UQTR) et membre associé de l'Institut de recherche sur les PME (INRPME). INRS, 385, rue Sherbrooke Est, Montréal (Québec) H2X 1E3 Canada kadiag.aka@ucs.inrs.ca

François LABELLE, Ph.D.

Professeur à l'École de Gestion de l'UQTR et directeur de l'INRPME. INRPME, 3351, boul. des Forges, C.P. 500, Trois-Rivières (Québec), Canada G9A 5H7 françois.labelle@uqr.ca

\begin{abstract}
Michel TRÉPANIER, Ph. D.
Professeur à l'INRS, professeur invité à l'École de Gestion de l'UQTR et membre associé de

l'INRPME.

INRS, 385, rue Sherbrooke Est, Montréal (Québec) H2X 1E3 Canada michel.trepanier@ucs.inrs.ca
\end{abstract}

\begin{abstract}
Résumé
Les enjeux de la durabilité offrent des possibilités considérables d'innovations durables (ID) aux entreprises. Pour en profiter, les dirigeants peuvent s'impliquer dans le développement de ces innovations en exerçant trois rôles essentiels : champion durable, entrepreneur durable et leader durable. L'approche par les traits utilisée pour révéler ces rôles est insuffisante pour comprendre comment les dirigeants parviennent à développer des ID dans différents contextes. Nous proposons une approche comportementale pour répondre à cette question tout en insistant sur la relation complémentaire entre les trois rôles. Au terme de l'exploration de deux cas d'ID développées par des PME, nos résultats montrent que pour réaliser ces innovations, les dirigeants de PME jouent les trois rôles et les intègrent en se comportant comme des observateurs actifs, des bricoleurs et des réseauteurs ainsi qu'en composant avec les événements constitutifs des contextes dans lesquels ils évoluent. Notre étude conclut en suggérant un modèle intégré qui présente les trois rôles et les comportements par lesquels ils sont activés comme les dimensions constituantes d'un construit unique : l'éco-innovateur.
\end{abstract}




\begin{abstract}
The sustainability issues offer considerable possibilities for sustainability-oriented innovations (SOI) to firms. To take advantage of it managers must be involved in the development of these innovations by playing three main roles : sustainable champion, sustainable entrepreneur and sustainable leader. The trait approach used to reveal these roles is insufficient to understand how managers succeed in developing SOI in various contexts. We propose a behavioral approach to answer this question while insisting on the complementary relationship between the three roles. At the end of the exploration of two case of SOI made by SMEs, our results show that to develop these innovations, SME's managers undertake the three roles by behaving as active observers, handyman and networkers as well as composing with the constitutive events of the contexts in which they evolve. Our study concludes by suggesting an integrated model which presents the three roles and the behaviors by witch they are enabled as the constituent dimensions of a single construct : the eco-innovator.
\end{abstract}

Keywords: SME - Sustainability - Innovation - Roles - Traits - Behaviors

\title{
Resumen
}

Las puestas de durabilidad ofrecen posibilidades considerables de innovaciones sostenibles (IS) a las empresas. Para sacar provecho de eso los dirigentes pueden implicarse tres papeles esenciales entre los que están los de campeón sostenible, de empresario sostenible y de líder sostenible. La aproximación por las rayas utilizada en la literatura para describir estos papeles es insuficiente para comprender cómo los dirigentes llegan a desarrollar IS en diferentes contextos. La exploración de dos casos IS desarrolladas en contexto de PYME permite ver que el enfoque comportamental que proponemos es adaptado más para ilustrar lo que hacen los dirigentes. Nuestros resultados muestran que para desarrollar IS, nuestros dirigentes de PYME juegan los tres papeles comportándose como observadores activos, personas mañosas y networkers. Nuestro estudio concluye sugiriendo un modelo integrado que presenta los tres papeles y los comportamientos subyacentes como las dimensiones constituyentes de construido único : el eco-innovador.

Palabras claves: PYME - Durabilidad - Innovación - Papeles - Tiros - Comportamientos 


\section{Introduction}

Les préoccupations croissantes à l'égard de la durabilitél constituent une force influente qui redéfinit les règles du jeu de la concurrence et la façon de développer les innovations (Tidd, Bessant et Pavitt, 2006). Dans ce contexte, "les entreprises qui sauront développer des innovations durables seront celles qui survivront » (Hall, 2002, p. 196, notre traduction). Les innovations durables (ID) ou les éco-innovations sont des produits, services ou procédés (ou une combinaison de deux d'entre eux, p. ex.: un produit-service) nouveaux ou améliorés et plus durables, des marchés nouveaux ou élargis respectueux de l'environnement, des nouveaux modèles commerciaux orientés vers la durabilité (OCDE, 2010). Les chercheurs se questionnent de plus en plus sur comment sont développées les ID dans les PME (Adams et al., 2016). Nous avons identifié trois lacunes dans la littérature liées à cette question et, dans ces conditions, notre étude vise trois objectifs.

Premièrement, bien que le dirigeant de PME soit considéré comme un acteur central dans le développement des ID (Bos-Brouwers, 2009), les rôles qu'il joue dans ce processus ont reçu très peu d'attention dans la littérature (Auteur, 2015). Porter et Kramer (2006) recommandent qu'on s'intéresse de plus en plus à ces rôles parce que les dirigeants doivent être stratégiques dans l'évaluation des enjeux de la durabilité et dans leur manière de les résoudre. Dans les milieux de pratique (p. ex.: au Québec) les dirigeants de PME veulent comprendre et apprendre davantage sur ce qu'ils doivent faire pour développer des ID (voir rapport du REDD, 2012). Compte tenu de ces préoccupations, notre étude vise à contribuer à l'avancement des connaissances sur les ID en s'intéressant aux rôles que jouent le dirigeant de PME dans leur développement.

Deuxièmement, quelques travaux identifient les rôles de champion durable (Anderson et Bateman, 2000), d'entrepreneur durable (Larson, 2000) et de leader durable (Egri et Herman, 2000) comme étant essentiels pour les dirigeants qui souhaitent développer des ID. Mais, ils le font selon une perspective présentant ces rôles comme le résultat de traits psychosociologiques : le dirigeant fait ceci (rôle) parce qu'il est cela (trait) (Triguero-Cano, Moréno-Mondejar et Davia, 2013). En cela, ces travaux s'appuient sur une approche par les traits qui a pourtant montré ses limites notamment pour comprendre comment l'entrepreneur développe une innovation (Bird, Schjoedt et Baum, 2012). Partant des trois rôles déjà identifiés dans la littérature, notre étude vise à combler les limites de l'approche par les traits en proposant une analyse s'appuyant sur une approche comportementale.

Troisièmement, dans la littérature les trois rôles sont présentés de façon éparse. Seul Bossink (2013) insiste sur leur relation complémentaire nécessaire pour développer des ID et propose ainsi une analyse intégrée de ces rôles. Pour l'auteur «Three distinctive individual roles or personal behaviors play a pivotal role in the [development of ideas to innovate in

\footnotetext{
${ }^{1}$ Le terme « durabilité » (tout comme le terme durable) fait allusion au développement durable (Bansal et Song, 2017). Selon les fondements du développement durable, tous les acteurs de la société doivent initier toute action qui lie des objectifs économiques à des enjeux environnementaux et sociaux de manière à permettre aux générations présentes et futures de répondre à leurs besoins. Ainsi, une personne, une action ou une activité est orientée vers la durabilité (ou est durable), lorsqu'elle valorise cette liaison entre des objectifs économiques, environnementaux et sociaux.
} 
sustainabilitty] : the leader, the entrepreneur, and the champion » (p. 4). Cependant, Bossink a développé sa réflexion à partir d'une étude du développement des ID dans les grandes entreprises et son analyse intégrée des rôles n'a pas été appliquée au contexte des PME. Dans les PME, le dirigeant tient simultanément plusieurs rôles (O'Gorman, Bourke et Murray, 2005) en raison de la petite taille de ce type d'organisation et des façons de faire en matière d'innovation (Rothwell et Dodgson, 1991). Par conséquent, il est fort probable que le dirigeant de PME joue à la fois les trois rôles à travers des comportements spécifiques au cours du développement d'une ID. En nous inspirant des travaux de Bossink, notre étude a pour objectif de faire une analyse intégrée des trois rôles selon une approche comportementale en contexte de PME.

Ce focus sur les rôles du dirigeant de PME dans le développement des ID selon une approche comportementale et une analyse intégrée présente plusieurs avantages. D'une part, une approche comportementale donne aux dirigeants de PME une connaissance plus pratique et réaliste de ce qu'ils peuvent faire pour développer une ID. Elle permet également à ces dirigeants de connaître certains comportements spécifiques à adopter dans diverses situations tout au long du développement d'une ID afin d'accroître ses possibilités de succès. D'autre part, en proposant une analyse intégrée, notre étude permet de présenter une vision systémique des trois rôles qui prend mieux et davantage en considération les dynamiques comportementales des dirigeants de PME, les caractéristiques propres des ID et les processus en vertu desquels celles-ci sont produites ainsi que le contexte dans lequel le tout se manifeste. Ce faisant, nous souhaitons répondre à la question spécifique suivante: Comment le dirigeant de PME concilie-t-il dans des comportements spécifiques les rôles de champion durable, d'entrepreneur durable et de leader durable afin de réaliser une ID ?

Pour répondre à cette question, nous spécifions d'abord le cadre théorique de notre étude. Ensuite, nous décrivons la méthodologie de recherche utilisée qui s'inscrit dans une logique interprétativiste. Dans la troisième partie, les résultats de l'étude sont présentés et analysés. Enfin, nous concluons par une discussion.

\section{Cadre théorique}

Dans cette partie nous définissons, premièrement, l'ID afin de cerner ses caractéristiques et certaines de ses implications pour le dirigeant de PME. Deuxièmement, nous décrivons l'essentiel des caractéristiques du dirigeant de PME telles qu'elles sont documentées par les deux approches (traits et comportements) utilisées dans les travaux sur la PME et l'entrepreneuriat. Troisièmement, nous montrons comment et avec quelles limites les trois principaux rôles identifiés dans la littérature sont analysés suivant l'approche par les traits. Quatrièmement, nous proposons une approche comportementale pour mieux comprendre ces rôles en insistant sur leur relation avec ce qui constitue le résultat des actions du dirigeant de PME (et son équipe), soit l'ID.

\subsection{Caractéristiques et implications des ID}

Fussler et James (1996) définissent pour la toute première fois les ID comme des innovations 
qui créent de la valeur pour les consommateurs et les entreprises tout en réduisant significativement les impacts environnementaux et sociaux négatifs. Ces innovations peuvent prendre la forme de procédé, produit, service (ou une combinaison de deux d'entre eux) ou de modèle d'affaires, nouveaux ou améliorés (OCDE, 2010). Les ID visent donc à résoudre des enjeux environnementaux et/ou sociaux tout en suivant des impératifs économiques, soit des enjeux de la durabilité (p. ex.: pollution atmosphérique, gestion des déchets, santé et sécurité des populations).

Pour Tidd et al. (2006), les enjeux de la durabilité offrent aux entreprises des opportunités considérables d'innovation mais également comportent des défis en matière de gestion. Les ID obligent les dirigeants à pratiquer une " gestion intégrée » parce qu'elles ont des « implications systémiques » et « découlent du souci et de la nécessité d'être en compatibilité avec les contextes sociaux, politiques et culturels complexes » (Ibid., p. 51). Selon Adams et al. (2016), l'ID « implique de transformer intentionnellement non seulement les produits, les procédés ou les pratiques mais également la philosophie et les valeurs d'une organisation [...] »(p. 180, notre traduction). Le développement des ID exige donc que les dirigeants adoptent de nouvelles façons de faire au risque de voir leurs ID échouer (Tidd et al., 2006).

Pour les organisations de petite taille, comme les PME qui ont pour avantages la souplesse et la prise de décision rapide mais pour désavantages des limites au niveau des ressources et compétences (Ibid.), l'adoption de ces nouvelles façons de faire repose en grande partie et directement sur le dirigeant de PME (Rothwell et Dodgson, 1991). Or, nous savons que les caractéristiques de ce dernier influencent ses décisions et ses façons de faire (Ibid.).

\subsection{Caractéristiques du dirigeant de PME}

Les travaux sur les caractéristiques du dirigeant de PME font état de deux approches: l'approche par les traits et l'approche comportementale. Notre objectif dans cette section n'est pas de présenter une recension exhaustive des écrits concernant ces approches. Il s'agit plutôt d'avoir un aperçu de ce qui les distingue « fondamentalement » et de voir les implications de ces distinctions sur le concept de rôle.

Les traits peuvent être définis comme les caractéristiques d'une personne (Stevenson et Jarillo, 1990) qui sont relativement stables quelle que soit la situation (Gartner, 1989) et qui prédisposent cette personne à réfléchir, sentir ou agir d'une certaine manière (Bandura, 2012). L'approche par les traits distingue les entrepreneurs des non-entrepreneurs à partir de trois catégories de traits psychosociologiques (Gartner, 1989): traits de personnalité (p. ex.: conscience, valeurs, ouverture), traits cognitifs (p. ex.: visionnaire, créatif, ambitieux, intelligent) et traits sociaux (p. ex.: genre, âge, éducation). Parce qu'en contexte de PME le dirigeant (tout comme l'entrepreneur) et son entreprise ne font souvent qu'un et que ce dernier en est un acteur central (Julien et Marchesnay, 1996), des travaux en entrepreneuriat considèrent que certains traits sont particuliers au dirigeant de PME et constituent un facteur explicatif de tout ce qu'il crée, développe ou entreprend (Verstraete et Saporta, 2006). Par exemple, la persistance dans l'action, le goût pour l'indépendance et l'autonomie sont caractéristiques du dirigeant de PME. 
Malgré son utilité, l'approche par les traits présente des lacunes. L'une des plus importantes selon Gartner (1989) porte sur le fait que cette approche ne peut à elle seule expliquer les décisions et les actions de l'entrepreneur parce que celui-ci doit aussi composer avec des éléments externes à lui tels que le contexte et les acteurs. Pour Gartner (1989) « [...] les approches par les traits ont été infructueuses et les approches comportementales présentent une perspective plus riche pour les recherches futures [...]»(p. 47, notre traduction). Autrement dit, il faut étudier les comportements pour mieux comprendre ce que fait l'entrepreneur et comment il le fait.

Bird et al. (2012) définissent le comportement comme " the concrete enactment by individuals (or teams) of tasks or activities [...], which are required in some combination to start and grow most new organizations » (Bird et al., 2012, p. 890). Le comportement est donc associé à l'activation d'actions, d'activités par un individu ou ses réponses aux stimuli du contexte dans lequel il évolue. Encourager la participation des employés, obtenir du soutien et des capitaux, communiquer avec les clients, concevoir des produits et services, répondre aux attentes des acteurs externes, sont des exemples de comportements qui ont pour particularité d'être visibles ou audibles contrairement aux traits (Gartner, 1989). Dans la littérature sur l'implication du dirigeant de PME dans le développement des ID, c'est sous le prisme des traits que les rôles de ce dernier sont révélés.

\subsection{Les rôles sous le prisme des traits}

Plusieurs traits sont attribués aux dirigeants lorsqu'il est question de leur implication dans le développement des ID. Afin de cerner de quelle manière l'implication ou les rôles du dirigeant de PME dans le développement des ID sont abordés sous le prisme des traits, les sections suivantes font état de trois principaux rôles présentés de façon éparse dans la littérature : le champion durable, l'entrepreneur durable et le leader durable.

Le champion durable est celui qui est attentif aux enjeux de la durabilité (Sandström et Tingström, 2008). Il a une vision positive de ces enjeux, est conscient de leur pertinence et est réceptif à leur égard (Gadenne, Kennedy et McKeiver, 2009). En clair, le champion durable est sensible aux enjeux de la durabilité parce qu'ils peuvent avoir une incidence sur les activités de son organisation ou sur des personnes qui lui sont proches. En contexte de PME, c'est très souvent le dirigeant qui joue le rôle d'un champion durable en raison de ses traits psychosociologiques (Bos-Brouwers, 2010; Jenkins, 2009). Ces traits peuvent être sociaux tels que l'âge, l'éducation, ou liés à sa personnalité tels que les valeurs et convictions environnementales ou sociales (Auteurs, 2010). Par exemple, étant donné la nature plus contemporaine des enjeux de la durabilité, les dirigeants de PME plus jeunes (Gadenne et al., 2009; Vives, 2006), ayant un niveau d'éducation plus élevé (Schaper, 2002) seraient des champions durables parce qu'ils sont plus sensibles à ces enjeux et, par conséquent, plus orientés vers les ID. En somme, compte tenu de ses traits, le champion durable est celui qui fait des enjeux de la durabilité son « cheval de bataille ».

L'entrepreneur durable est, en termes de traits cognitifs, celui qui a une vision ou une ambition environnementale; il est créatif et a l'intention d'innover en intégrant des enjeux environnementaux (Berchicci, 2005). En termes de traits de personnalité, il est ouvert aux 
opportunités qu'il perçoit à travers les enjeux de la durabilité (Parker et al., 2009). Il le fait pour différentes raisons : pour se soumettre aux règlementations environnementales, pour en tirer des avantages financiers ou parce qu'il épouse des causes environnementales et sociales. Dans une logique de croissance, l'entrepreneur durable est plus sensible à tout ce qui lui permettrait de faire croitre son entreprise (Larson, 2000). Ainsi les ID sont perçues par ce dernier comme une opportunité de créer de la valeur durable (Moore et Manring, 2009). En somme, l'entrepreneur durable est une personne ouverte aux opportunités liées à des enjeux de la durabilité.

Le leader durable est celui qui est extraverti envers les gens, soit sociable, chaleureux, confiant, expressif, volontariste, motivé, etc. (Hansen et al., 2002; Walker et al., 2008). Pour Bos-Brouwers (2009), le leadership durable est un des traits caractéristiques du dirigeant de PME orienté vers les ID. En tant que tel, il est sensible aux demandes et préoccupations des parties prenantes impactées par les incidences environnementales et sociales de ses activités. Dans ce sens, il demeure une personne ouverte et attentive aux autres : l'empathie est donc un de ses traits. Selon Jenkins (2009), « il est essentiel pour les dirigeants de PME d'exprimer un fort leadership, s'ils veulent promouvoir des valeurs environnementales et sociales au sein de leur organisation » (p. 34, notre traduction). En somme, le leader durable présente très souvent les traits d'une personne orientée vers les relations interpersonnelles.

Les trois rôles que nous venons de présenter sont décrits comme des traits psychosociologiques. Dans l'approche par les traits, on retrouve une causalité implicite et un peu naïve voulant que si une personne possède les traits « $\mathrm{x}$ », elle jouera le rôle « $\mathrm{y}$ » approprié. Nous soutenons que pour comprendre comment le dirigeant de PME parvient à développer une ID, il faudrait mobiliser une approche comportementale des rôles. L'approche comportementale reprend, par ailleurs, la conception du rôle du dirigeant par les activités telle que développée par Carlson (1951), ensuite popularisée par Mintzberg (1984). Chez ces auteurs, le rôle est émergent dans la mesure où le dirigeant l'exerce dans un but précis et à travers des comportements en enchainant des décisions qu'il prend "dans l'action » en fonction des évènements et de ses interactions avec d'autres acteurs (Desmarais et Abord de Chatillon, 2010). Cependant, le recours à l'approche comportementale ne doit pas faire oublier que « comme pour l'école des traits de personnalité, il manque à ce courant de pensée une relation de type complémentaire [...] entre l'individu entrepreneur et ce qui constituera le résultat de son activité » (Verstraete et Saporta, 2006, p. 92). C'est pourquoi, sous le prisme du comportement nous nous intéressons également à la relation complémentaire entre les trois rôles puis entre ceux-ci et l'ID.

\subsection{Les rôles sous le prisme du comportement}

En soutenant une approche comportementale, nous tenons compte des critiques à l'encontre de l'approche par les traits exprimées par des chercheurs dans les champs de la PME et de l'entrepreneuriat, sans pour autant en rejeter la pertinence. Nous nous inspirons également des travaux de Bossink (2013) qui insiste sur la relation complémentaire entre les trois rôles, nécessaire pour développer des ID. Dans les sections qui suivent nous définissons d'abord le concept de rôle étroitement lié au comportement. Ensuite, nous proposons une analyse des trois rôles selon une approche comportementale. 


\section{Définition du concept de rôle}

Pour Biddle (1986), les comportements sont une expression du rôle qui peut être défini de trois manières : structurelle, individuelle ou interactionniste. De manière structurelle, les rôles sont définis comme les comportements d'une personne occupant un poste ou une fonction au sein d'un système social stable et qui consistent à activer un ensemble de tâches obligatoires (Fondas et Stewart, 1994). Dans cette perspective, le rôle est « écrit » à l'avance et attribué à une personne. Il est ainsi une émanation de la structure formelle. Et, le rôle du dirigeant est alors la simple mise en œuvre de ce qui est « prévu dans et par le script » correspondant à sa fonction (Fayol, 1916 ; Linton, 1945 ; Parsons, 1954 ; cités dans Desmarais et Abord de Chatillon, 2010). Cette définition a été critiquée pour son ancrage déterministe du rôle et pour sa description du dirigeant comme un acteur passif (Wrzesniewski et Dutton, 2001). Dans leur dimension individuelle, les rôles du dirigeant sont des réponses aux attentes qui lui sont exprimées par des personnes à l'intérieur comme à l'extérieur de l'organisation (Fondas et Stewart, 1994). Contrairement à ces deux définitions, la définition interactionniste permet plutôt de considérer que les dirigeants ne sont pas simplement des marionnettes dans les mains de ceux qui tirent les ficelles et déterminent leurs comportements (Biddle, 1986). Dans la perspective interactionniste, les rôles sont produits sur une base volontaire par des comportements qui eux-mêmes résultent des actions réciproques entre des individus interagissant dans un contexte qu'ils partagent et qui influencent les actions de chacun. Ici, le rôle comporte une dimension à la fois structurelle et individuelle. La définition interactionniste a pour avantage de présenter une vision dynamique et systémique du rôle. Elle rejoint, de ce fait, l'approche émergente développée par Mintzberg dans la foulée de Carlson (Desmarais et Abord de Chatillon, 2010). C'est sur la base de la définition interactionniste du rôle que nous proposons, dans ce qui suit, une analyse intégrée des rôles de champion durable, d'entrepreneur durable et de leader durable.

\section{Le rôle de champion durable}

Le champion durable cherche à identifier des enjeux de la durabilité et à les lier de manière concrète au futur de son organisation. Son rôle porte sur l'identification, l'interprétation et la sensibilisation aux enjeux de la durabilité auxquels fait face son organisation (Anderson et Bateman, 2000). En effet, l'identification des enjeux de la durabilité consiste à " scanner » l'environnement (interne et externe) de l'entreprise afin d'y repérer des informations significatives à partir de diverses sources (p. ex.: employés, contacts externes, rapports publics). Le champion durable interprète ensuite les informations liées à ces enjeux en insistant sur les incidences qu'elles comportent pour l'organisation (Banerjee, 2001; Sharma, 2000). Enfin, il sensibilise différents acteurs internes et externes à ces incidences potentielles en leurs fournissant des informations pertinentes (Yukl, 2001). En somme, le champion durable « recherche des tendances, traite des informations, partage des connaissances qui peuvent générer de nouvelles idées » (Bossink, 2013, p. 5) en lien avec des enjeux de la durabilité. C'est à partir de ces tendances, informations ou connaissances qu'il peut finalement découvrir une opportunité d'ID et ainsi activer son rôle d'entrepreneur durable en les saisissant et en les transformant en projet d'ID.

\section{Le rôle d'entrepreneur durable}

L'entrepreneur durable cherche à résoudre des enjeux de la durabilité en explorant ou en 
exploitant, par le développement d'une ID, les opportunités qu'ils comportent (York et Venkataraman, 2010). En ce sens, pour Schaltegger et Wagner (2010), un entrepreneur durable se distingue d'un entrepreneur « classique », parce qu'il construit « des ponts » entre des enjeux de la durabilité et les besoins du marché. En effet, pour Patzelt et Shepherd (2010), les ID développées par un entrepreneur durable sont l'aboutissement d'un processus qui débute par la reconnaissance d'opportunités d'ID, soit « des opportunités qui visent non seulement à améliorer l'écosystème mais également à procurer des retombées économiques, environnementales et sociales aux humains »(p. 2, notre traduction). Ces opportunités trouvent leurs sources tant dans les défaillances du marché (Dean et McMullen, 2007) que dans la connaissance qu'a l'entrepreneur des enjeux de la durabilité (York et Venkataraman, 2010). Ainsi, plus l'entrepreneur est au fait de ces défaillances et possède une bonne connaissance de ces enjeux, plus il sera susceptible de développer des ID (Patzelt et Shepherd, 2010). Pour Bossink (2013), dans son rôle d'entrepreneur durable, le dirigeant est celui qui « voit où se présentent de nouvelles opportunités de vendre des produits, détecte où se cachent les possibilités de nouveaux services, et crée des projets ou des entreprises pour lancer ces produits et services, et ouvrir des marchés » (p. 4, notre traduction). En somme, le rôle d'entrepreneur durable consiste à activer des comportements axés sur la reconnaissance, la saisie d'opportunités d'ID et finalement la définition d'un projet d'ID. Pour transformer le projet d'ID en ID, il devra cependant activer le rôle de leader durable afin d'acquérir les ressources et les compétences qui lui sont nécessaires mais lui font défaut.

\section{Le rôle de leader durable}

Shrivastava (1994) indique que, pour le leader durable les relations sociales sont étroitement liées à tout projet visant des enjeux de la durabilité et que, partant de là, les interactions sociales sont au centre de ses actions. Dans ce sens, il va à la rencontre de différentes parties prenantes, élabore des compromis et cherche à acquérir des ressources afin de répondre à ces enjeux. Ce faisant, il doit avoir « la capacité d'influencer des personnes et de les mobiliser autour d'une vision commune et à long terme des enjeux environnementaux [...]» (Egri et Herman, 2000, p. 572, notre traduction) et des solutions à y apporter. Dans ce sens, pour Egri et Frost (1994), le rôle de leader durable « n'est pas une action isolée, mais il consiste à développer des collaborations à l'intérieur comme à l'extérieur de l'organisation » (p. 199, notre traduction). Dans cette démarche de collaboration, la capacité de mobilisation du leader durable va lui permettre d'apporter une réponse aux demandes des parties prenantes et, par conséquent, de légitimer ses projets. Bossink (2013) précise que le rôle de leader durable en particulier dans un projet d'ID, consiste à « [...] avoir une vue d'ensemble des parties prenantes de la firme ainsi que de leurs demandes et préoccupations qu'il tente de résoudre en se souciant de l'équilibre entre les intérêts de ces derniers » (p. 24, notre traduction). En somme, le rôle de leader durable consiste à activer des comportements axés sur les relations interpersonnelles tant à l'intérieur qu'à l'extérieur de l'entreprise.

Il faut souligner que c'est la complémentarité entre les trois rôles que nous venons de décrire qui permet de réaliser l'ID. C'est sur la base de cette approche comportementale des rôles que nous souhaitons répondre à notre question de recherche : comment le dirigeant de PME concilie-t-il dans des comportements spécifiques les rôles de champion durable, d'entrepreneur durable et de leader durable afin de réaliser une ID ? Pour ce faire, nous avons mené une étude de cas multiples s'appuyant sur une démarche qualitative et une posture 
interprétativiste.

\section{Méthodologie}

La présente étude s'appuie sur une recherche qualitative qui se prête bien aux études sur les ID dans les PME, parce qu'elle permet de faire une analyse approfondie des pratiques et des comportements des dirigeants de PME en lien avec les ID (Klewitz et Hansen, 2014). En nous inscrivant dans une logique interprétativiste, nous voulons appréhender les actions des dirigeants de PME à partir de leurs propres points de vue (Thiétart, 2007) puisque ce sont eux qui activent ces comportements à travers lesquels ils « font exister » leurs ID. Pour tenir compte des spécificités de la PME, la littérature sur les ID utilise en plus des critères quantitatifs (p. ex.: moins de 200 employés, selon l'Institut de la Statistique du Québec), des critères qualitatifs ayant trait aux forces et faiblesses de celle-ci. Ainsi, la PME est définie comme une entreprise dont, entre autres, la flexibilité organisationnelle et la position centrale ainsi que l'orientation (p. ex.: sensibilité, valeurs et convictions par rapport aux enjeux de la durabilité) et l'implication directe du dirigeant, sont des forces favorisant le développement des ID (Bos-Brouwers, 2009). Elle est également décrite comme une entreprise dont les ressources limitées constituent des faiblesses empêchant le dirigeant de s'engager dans le développement des ID (Brio et Jùnquera, 2003). C'est sur la base de critères qualitatif et qualitatif (orientation du dirigeant et son implication directe qui le qualifie pour présenter le récit de son ID et pour témoigner du sens de son propre rôle ainsi que de l'expérience perçue chez d'autres acteurs.), que les PME parmi lesquelles nous allions sélectionner nos cas ont été identifiées.

\subsection{Sélection des cas}

Les cas ont été sélectionnés dans un échantillon qualitatif qui présente les caractéristiques suivantes. Premièrement, il comporte des cas multiples qui permettent d'étudier les rôles et comportements des dirigeants de PME dans une variété de contextes, assurant ainsi des résultats plus riches (Yin, 2003). Deuxièmement, il implique la sélection d'un petit nombre de cas qui favorise une étude en profondeur (Miles et Huberman, 2003). Troisièmement, l'échantillon qualitatif est souvent conditionné par un opportunisme méthodologique qui consiste à identifier des cas donnant l'opportunité d'étudier le phénomène visé et à négocier l'entrée du chercheur sur le terrain (Girin, 1990). Cet opportunisme doit être cependant contrôlé par des critères spécifiés à l'avance (Miles et Huberman, 2003). Partant de là, deux cas de PME ont été retenus de la façon suivante. D'abord, pour identifier les cas potentiels, nous avons eu recours aux répertoires (en ligne) de deux organisations québécoises EnviroAccess et Novae - qui font la promotion de dirigeants et de PME de différents secteurs d'activités qui développent des ID. Ensuite, pour négocier notre entrée sur le terrain, nous avons contacté par courriel les dirigeants de $35 \mathrm{PME}$ dont 20 respectaient les critères choisis plus haut. Parmi les 20 PME, nous avons retenu deux qui avaient respectivement 22 et 115 employés. Ces deux PME présentaient l'avantage d'être immédiatement disponibles, de répondre à tous nos critères en plus d'être représentatifs des considérations théoriques faisant l'objet de cette recherche. C'est avec les deux principaux dirigeants de ces PME (soit la cosméticienne équitable et le chimiste durable) que nous avons fait des entretiens semi- 
dirigés.

\subsection{Entretiens avec les dirigeants}

Les récits des dirigeants ont été recueillis au moyen d'entretiens préliminaires et en profondeur. Les premiers, d'une durée de 30 minutes, ont été réalisés par téléphone avec chaque dirigeant. Ils visent à amener les dirigeants à mieux comprendre l'objet d'étude et à les préparer aux entretiens en profondeur. Avec chaque dirigeant, l'entretien en profondeur a duré environ deux heures. Une question est posée au dirigeant: " racontez-nous dans les détails l'histoire du développement de votre ID? Nous « laissons parler » le dirigeant qui présente et, d'une certaine manière, " explique » (souvent sous l'impulsion de questions de précision) ses actions et ses interactions avec d'autres acteurs dans ses propres mots et en fonction de son propre point de vue. Son récit n'est pas construit par et à travers les catégories du chercheur. Par ailleurs, le caractère confidentiel des projets d'ID n'a pas permis d'interroger " directement» des acteurs externes impliqués dans ces processus tels que les clients, les fournisseurs ou les partenaires d'affaires. Il a été retenu ce que les deux dirigeants et leurs collaborateurs (le vice-président, un ami de la cosméticienne équitable ainsi que des collaborateurs du chimiste durable, soit une coordonnatrice en développement durable, un directeur technique et un vice-président d'un département de l'entreprise) rapportent sur les expériences de ces derniers. De plus, d'autres sources de données telles que des documents (une trentaine) privés obtenus de l'entreprise (prospectus de présentation, des rapports d'activités) et publics provenant d'archives de presse locale ont été utilisés. Ces documents ainsi que les entretiens avec les collaborateurs ont permis de s'assurer de la crédibilité des récits des deux dirigeants (Van de Ven, Angle et Poole, 2000).

\subsection{Analyse des données}

L'analyse des données issues des entretiens consiste à étudier le contenu des processus sociaux ayant conduits au développement des ID à l'intérieur desquels les rôles et les comportements des dirigeants émergent. Dans l'analyse de contenus de processus, c'est la présentation détaillée des actions des acteurs et des évènements qui permet de mettre en évidence les comportements qu'ils assument au travers des interactions qu'ils entretiennent jusqu'à la réalisation de l'objet visé (Van de Ven et al. 2000). Une grille d'analyse (Tableau 1) a été élaborée afin de permettre une analyse de contenus systématique avec le logiciel Nvivo. Cette grille comprend les thèmes ou nœuds (en langage Nvivo), leur définition ou mémos (qui nous orientent tout au long de l'analyse) et les termes sensibilisants ou référents (que l'on retrouve dans les extraits significatifs - phrases ou paragraphes - se rapportant aux thèmes) qui, ici, sont les comportements des interviewés.

\section{Tableau 1: Grille d'analyse des données}

\begin{tabular}{lll}
\hline Thèmes & Définition & Exemples de termes sensibilisants \\
\hline
\end{tabular}




\begin{tabular}{|c|c|c|}
\hline Champion durable & $\begin{array}{l}\text { Actions, activités ou réponses aux } \\
\text { stimuli de l'environnement visant à } \\
\text { rechercher ou acquérir des } \\
\text { informations liées à des enjeux de } \\
\text { durabilité prioritaires à la fois pour } \\
\text { l'entreprise et pour la société }\end{array}$ & $\begin{array}{l}\text { Rechercher des tendances } \\
\text { Traiter des informations } \\
\text { Partager des connaissances } \\
\text { Identifier des enjeux de la durabilité } \\
\text { Interpréter l'information } \\
\text { Utiliser l'information pour agir }\end{array}$ \\
\hline Entrepreneur durable & $\begin{array}{l}\text { Actions, activités ou réponses aux } \\
\text { stimuli de l'environnement visant à } \\
\text { reconnaitre et saisir des opportunités } \\
\text { d'ID et les transformer en projet d'ID }\end{array}$ & $\begin{array}{l}\text { Explorer ou exploiter des opportunités } \\
\text { Répondre aux besoins du marché } \\
\text { Repérer des opportunités d'ID } \\
\text { Transformer les opportunités en ID } \\
\text { Créer des projets ou une entreprise } \\
\text { Lancer un produit, un service ou un } \\
\text { procédé } \\
\text { Ouvrir des marchés }\end{array}$ \\
\hline Leader durable & $\begin{array}{l}\text { Actions, activités ou réponses aux } \\
\text { stimuli de l'environnement visant à } \\
\text { développer des relations } \\
\text { interpersonnelles à l'intérieur comme à } \\
\text { l'extérieur de l'entreprise }\end{array}$ & $\begin{array}{l}\text { Acquérir des ressources et compétences } \\
\text { Influencer des personnes } \\
\text { Mobiliser autour d'une vision commune } \\
\text { Développer des collaborations } \\
\text { Légitimer des actions, des projets } \\
\text { Confier des rôles } \\
\text { Impulser les initiatives } \\
\text { Élaborer des compromis } \\
\text { Identifier, contacter, recruter des } \\
\text { personnes et des organisations }\end{array}$ \\
\hline Évènement & $\begin{array}{l}\text { Fait déterminant, incident, point } \\
\text { critique au cours duquel se produisent } \\
\text { les actions, les activités ou les réponses } \\
\text { aux stimuli de l'environnement }\end{array}$ & $\begin{array}{l}\text { Rencontre critique mais fortuite } \\
\text { Incident } \\
\text { Problème } \\
\text { Intervention extérieure }\end{array}$ \\
\hline
\end{tabular}

Pour s'assurer d'une analyse systématique, les entretiens ont été transcrits sous forme de verbatims puis transférés dans un support informatique unique qui facilite leur organisation et leur manipulation (Gauthier et al., 2006). L'un des chercheurs a procédé avec le logiciel Nvivo à un codage des extraits significatifs. Ensuite, pour assurer la fiabilité des analyses et toujours à partir de la grille d'analyse du tableau 1, le codage des extraits significatifs suggérés par le chercheur 1 a été discuté et questionné par deux autres chercheurs afin d'en vérifier la pertinence, et d'aboutir à un accord (Thiétart, 2017). Dans la section qui suit, les deux cas d'ID sont exposés en présentant les deux principaux dirigeants (soit la cosméticienne équitable et le chimiste durable) et le contexte ayant suscité leurs actions puis nous décrivons leurs ID.

\subsection{Présentation des cas}

La présentation des cas nous situe le contextes dans lequel les dirigeants ont évolué et les ID ont été développées. Nous y verrons deux dirigeants de PME sensibles à des enjeux de la durabilité et ayant bénéficié du soutien de différents acteurs qui leur ont permis de réaliser les ID.

\section{La cosméticienne équitable}

En 2004, dans le cadre d'un stage de coopération internationale, celle qui sera plus tard à la tête d'une PME de 22 employés créée en 2007 et qui évolue dans le secteur du cosmétique, se 
retrouve à travailler auprès d'une coopérative de femmes africaines produisant de l'huile d'argan. Le hasard a voulu qu'elle soit logée chez une de ces femmes. Pendant son séjour, le propriétaire des terres sur lesquelles ces dernières cultivent l'arganier (arbre produisant l'huile d'argan) s'est plaint de ne pas avoir reçu les derniers paiements de loyer. De plus, pour lui, la vente de ses terres à une multinationale serait plus rentable. Les femmes sont donc menacées d'expulsion. Touchée par cet évènement « malheureux » qui met à mal le bien-être socioéconomique de ces femmes (enjeu social), la dirigeante décide de faire quelque chose pour les aider : " j'ai donc acheté toute leur production d'huile d'argan. Mais, pendant plusieurs mois, je ne savais pas quoi faire avec ce stock d'huile une fois revenue au Québec ». Grâce à des biochimistes qu'elle rencontre par l'intermédiaire d'un de ses amis (avec lequel elle s'associe par la suite et qui deviendra le vice-président), autrefois militant écologiste, la dirigeante comprend l'opportunité d'affaires que représente l'huile d'argan dans une perspective de développement durable. Pour elle, l'huile est une « ressource » naturelle et renouvelable, exploitée de manière durable tant au plan environnemental que social. Partant de là, et avec l'implication de ces biochimistes, d'une cosmétologue indépendante, des femmes productrices d'huile d'argan, et plus tard, de deux organismes de certification de produits équitables, la dirigeante parvient à coordonner (entre 2005 et 2007) le développement d'un cosmétique certifié écologique et équitable. Le cosmétique se présente comme une combinaison de produit et de service durables. En ce qui concerne le produit, il est fabriqué à partir d'une matière naturelle (l'argan) achetée directement auprès des femmes dans un pays d'Afrique. Dans ce pays, l'arganeraie est en voie de disparition (enjeu environnemental) et que, conséquemment, la région et l'arbre en tant que tel se sont vus reconnaître le statut de Réserve de la Biosphère, étant donné leur rôle primordial dans la lutte contre la désertification. L'huile d'argan est ensuite transformée au Québec pour obtenir une molécule sans recourir aux procédés utilisés pour la fabrication des cosmétiques synthétiques (« dérivés du pétrole et par conséquent dangereux pour la santé à long terme ", selon la dirigeante). Le cosmétique est enfin distribué dans des emballages faits de polymères de maïs biodégradables et compostables, et accompagnés d'un dépliant sur papier recyclé . En ce qui a trait au service, par des réductions ou des primes, les clients sont incités à rapporter les contenants usagés. La PME envoie une partie de ces contenants à une coopérative au Québec qui les utilise pour fabriquer des bijoux et réutilise l'autre partie.

\section{Le chimiste durable}

À la tête de plusieurs divisions importantes d'une entreprise de 115 employés créée en 1965 et qui évolue dans le secteur du traitement des eaux usées industrielles, le dirigeant entreprend en 2007 une mission d'observation en France afin d'en apprendre davantage sur les enjeux dans son secteur. Dans ce pays dont il est originaire et où, dès l'enfance, il a été sensibilisé aux enjeux environnementaux par son père, plusieurs épidémies causées par une bactérie qui se développe dans les eaux usées industrielles issues d'installations techniques telles que les spas, piscines et systèmes de climatisation centrale et qui ont provoqué plusieurs cas de décès, se sont produites. "Grâce à cette mission, j'ai acquis beaucoup de connaissances et d'expérience en ce qui concerne la bactérie à l'origine de ces épidémies. À la faveur de ces expériences, je décide d'ajouter le terme "durable" à mon titre de vice-président innovation pour montrer que c'est une de mes préoccupations et la nouvelle orientation que doit prendre l'entreprise ». Cette nouvelle orientation va conduire le dirigeant à développer une solution préventive avec l'implication d'acteurs internes, dont un directeur technique et des techniciens 
de laboratoires, permettant de prévenir l'apparition de la bactérie. En 2011, avec le soutien d'une association liée à la profession (dans le secteur d'activités), le dirigeant va à la rencontre des députés afin de les inciter à promulguer une loi qui préviendra l'apparition de la bactérie par un traitement préventif. Mais ces derniers refusent prétextant qu'il ne faut pas effrayer la population avec ce qui n'existe pas encore et qu'il existe déjà une solution standard que les intervenants du secteur (concurrents, services de santé publique, Régie du bâtiment) connaissent bien et utilisent depuis plusieurs années. Il faut signaler que cette solution s'attaque à la bactérie au moyen de produits toxiques et met deux semaines pour fournir les résultats du dépistage de la bactérie. Elle montrera ses limites en 2012 lors d'une crise qui surviendra au Québec et entraînera plusieurs décès. Contrairement à la solution standard, la solution préventive développée par l'entreprise (et donc bien avant la crise) se présente comme une combinaison de procédé et de produit durables. Elle comprend une technologie d'analyse et de dépistage en 24 heures ainsi qu'un programme de désinfection (procédé) par des produits écologiques. Dans la foulée de la crise de 2012, les services de santé publique, la Régie du bâtiment, les députés, l'association professionnelle et d'autres acteurs (chercheurs, populations touchées et médias) seront au centre d'une démarche qui conduira à l'élaboration d'une nouvelle loi obligeant les entreprises à procéder au contrôle de leurs installations techniques. Cette loi sera déterminante pour l'acceptation de la solution « durable et préventive ».

En somme, les ID ont été développées dans des situations et des circonstances différentes qui ont eu un impact sur les rôles et les comportements des dirigeants de PME que nous analysons dans la partie suivante.

\section{Résultats}

Nous débutons l'analyse des résultats en présentant dans le tableau 2 un portrait des principales actions des dirigeants dans le processus de développement des ID. Ici, les rôles ne sont pas un ensemble de fonctions prescrites ou déterminées par les traits des dirigeants étudiées. Les récits recueillis montrent plutôt qu'il s'agit d'actions (p. ex.: identifier, exploiter, mobiliser), autrement dit de comportements qui prennent forme et s'expliquent dans le cadre d'interactions contextualisées.

Tableau 2: Principales actions des dirigeants de PME dans le développement des ID

\begin{tabular}{|c|c|c|c|c|c|c|}
\hline \multicolumn{7}{|c|}{ Cosméticienne équitable } \\
\hline Actions & $\begin{array}{l}\text { Identifie un } \\
\text { enjeu de } \\
\text { durabilité: } \\
\text { bien-être } \\
\text { socio- } \\
\text { économique } \\
\text { des femmes }\end{array}$ & $\begin{array}{l}\text { Décide de } \\
\text { résoudre le } \\
\text { problème en } \\
\text { rachetant les } \\
\text { stocks d'huile } \\
\text { d'argan }\end{array}$ & $\begin{array}{l}\text { Contacte } \\
\text { une firme de } \\
\text { biochimistes }\end{array}$ & $\begin{array}{l}\text { Mobilise une } \\
\text { cosmétologue }\end{array}$ & $\begin{array}{l}\text { Sollicite deux } \\
\text { organismes de } \\
\text { certification } \\
\text { de produits } \\
\text { équitables }\end{array}$ & $\begin{array}{l}\text { Réalisation } \\
\text { du } \\
\text { cosmétique } \\
\text { écologique }\end{array}$ \\
\hline
\end{tabular}




\begin{tabular}{|c|c|c|c|c|c|c|}
\hline Évènements & $\begin{array}{l}\text { Expulsion } \\
\text { des femmes } \\
\text { des terres } \\
\text { d'arganeraie }\end{array}$ & & $\begin{array}{l}\text { Découverte } \\
\text { d'une } \\
\text { opportunité }\end{array}$ & & & et équitable \\
\hline Période & 2004 & & 2005 & & 2006 & 2007 \\
\hline \multicolumn{7}{|c|}{ Chimiste durable } \\
\hline Actions & $\begin{array}{l}\text { Identifie des } \\
\text { enjeux de } \\
\text { durabilité: } \\
\text { pollution de } \\
\text { l'air par la } \\
\text { bactérie, santé } \\
\text { et sécurité des } \\
\text { populations }\end{array}$ & $\begin{array}{l}\text { Décide } \\
\text { d'exploiter } \\
\text { l'opportuni } \\
\text { té au } \\
\text { Québec }\end{array}$ & $\begin{array}{l}\text { Forme une } \\
\text { équipe } \\
\text { interne }\end{array}$ & $\begin{array}{l}\text { Mobilise une } \\
\text { association } \\
\text { liée à la } \\
\text { profession } \\
\text { Rencontre les } \\
\text { députés }\end{array}$ & $\begin{array}{l}\text { Intervient } \\
\text { dans les } \\
\text { médias } \\
\text { Collabore } \\
\text { avec les } \\
\text { services de } \\
\text { santé et la } \\
\text { Régie du } \\
\text { bâtiment }\end{array}$ & \multirow[t]{2}{*}{$\begin{array}{l}\text { Acceptation } \\
\text { de la } \\
\text { solution } \\
\text { préventive }\end{array}$} \\
\hline Évènements & $\begin{array}{l}\text { Découverte de } \\
\text { la présence de } \\
\text { bactéries }\end{array}$ & & & $\begin{array}{l}\text { Refus des } \\
\text { députés de } \\
\text { voter une loi }\end{array}$ & $\begin{array}{l}\text { Apparition de } \\
\text { la bactérie au } \\
\text { Québec }\end{array}$ & \\
\hline Période & 2007 & & 2009 & 2011 & 2012 & 2013 \\
\hline
\end{tabular}

Dans chaque cas, le récit met de l'avant les actions des dirigeants. Et ce faisant, il montre que le développement des ID implique de la part de ces dirigeants des comportements qui sont en fait des interactions sociales elles-mêmes en lien direct avec les différents évènements auxquels ils sont confrontés à des périodes distinctes constituant chacune un contexte particulier. L'évolution de ces comportements montre également un changement de rôles. Dans les trois prochaines sections chacun de ces rôles est décrit en détail en prenant soin de mettre en évidence leur complémentarité et leur particularité dans le contexte des PME étudiées.

\subsection{Le champion durable}

L'analyse des données permet de voir qu'en raison de leurs convictions personnelles en matière de durabilité, de leur position centrale dans l'organisation, mais aussi grâce à d'autres acteurs avec lesquels ils interagissent (p. ex.: les biochimistes, l'équipe interne), les dirigeants de notre étude sont les personnes les mieux placées pour faire le lien entre les enjeux de la durabilité et leur ambition (dans le cas de la cosméticienne) ou la capacité interne de leur organisation à répondre à ces enjeux (dans le cas du chimiste).

Par exemple, dans le cas de la cosméticienne, le rôle de championne durable se manifeste en 2004 lorsque la future présidente reçoit l'information de l'expulsion des femmes grâce à l'une d'entre elles. Elle ressent et interprète cette information comme un enjeu social (bien-être socioéconomique de ces femmes) qu'il faut résoudre. Elle va acquérir par la suite plus d'informations et de connaissances sur la pertinence de cet enjeu en se rapprochant de la réalité vécue par ces femmes. 
«Puisque ces femmes étaient menacées d'expulsion, ma curiosité et mon envie de mieux comprendre ce qui leur arrive m'ont amenée à aller les voir sur leur lieu de travail. Quand j'ai vu ces 70 femmes de 30 à 99 ans assises par terre, fredonnant des chants traditionnels, occupées à casser des noix d'arganier pour en faire de l'huile d'argan, j'ai été touchée. Je me suis dit qu'il fallait faire quelque chose pour ne pas qu'elles soient expulsées, car cela aurait un impact négatif sur leur bien-être socioéconomique, puisque les revenus générés de cette activité permettent à ces femmes de subvenir à leurs besoins, d'assurer l'éducation de leurs enfants et d'avoir une dignité »".

Dans le cas du chimiste, ce dernier active son rôle de champion durable en 2007, lors d'une mission d'information et d'exploration en France. Cette mission permet au dirigeant (qui occupe une position centrale dans son organisation puisqu'il est à la tête de plusieurs divisions) d'acquérir, seul ou auprès d'anciens collègues, des informations et des connaissances au sujet de la présence d'une bactérie dans les tours de refroidissement. Le dirigeant découvre que la pollution atmosphérique (enjeu environnemental) liée à la diffusion de la bactérie dans l'air est à l'origine de plusieurs crises de santé publique (enjeu social) ayant entrainé des décès dans des pays d'Europe. Outre ses interactions avec des intervenants français, sa familiarité avec le contexte culturel (il est d'origine française) et technique (les eaux usées et les tours de refroidissement) français l'aide alors à percevoir la pertinence de cet enjeu au Québec et à cerner le réseau d'acteurs et d'objets techniques qu'il devra mettre en place pour développer son ID.

« J'ai donc mené des recherches plus poussées sur des cas de bactéries qui se sont produits en Europe et en Australie, et surtout sur les législations en vigueur dans des pays de ces deux régions. Puis, j'ai aussi joué une carte. Je me suis dit que le Québec et la France sont quand-même proches. Lorsqu'il y a des lois, des règlements qui sont pris en France, le Québec a tendance à s'en inspirer. Par exemple, le BNQ21000 est une norme de développement durable au Québec qui s'est beaucoup inspiré des normes en France et en Europe. De plus, au Québec, on retrouve de nombreux spas, systèmes de climatisation centrale, piscines utilisant des tours de refroidissement à l'eau propices au développement de la bactérie. J'ai donc pensé que cette bactérie pouvait aussi apparaitre ici ».

En somme, lorsqu'ils identifient, seuls mais le plus souvent avec d'autres acteurs, une information liée à des enjeux de la durabilité pouvant avoir une incidence sur leurs activités présentes (dans le cas de la Chimie durable) ou futures (dans le cas du Cosmétique durable), les dirigeants de PME décident que leur organisation doit agir pour trouver une solution. Par ailleurs, les analyses permettent de noter quelques particularités au sujet du rôle de champion durable dans le contexte des PME étudiées.

En effet, ce rôle est lié à la capacité des dirigeants de PME à déceler dans les informations recueillies un enjeu de la durabilité à partir d'une conjoncture présente (l'expulsion des femmes) ou passée (les épidémies en Europe) qu'ils vont ensuite interpréter. Dans ce sens, nous dirons que ces dirigeants se comportent comme des observateurs actifs de leur environnement. C'est dans les activités de cueillette et de traitement de l'information qui elles-mêmes se déploient dans des interactions contextualisées qu'ils font exister le rôle de champion durable. Dans le cas de la cosméticienne son comportement d'observatrice débute par une action réactive alors qu'elle est plutôt proactive chez le chimiste. Chez la 
cosméticienne, le comportement d'observatrice active se produit lorsque dans le contexte où elle vit (chez l'une des femmes), elle apprend d'abord la situation problématique vécue par les femmes et réagit ensuite en décidant de « faire quelque chose » : racheter tout le stock d'huile d'argan. Et, c'est seulement au contact des biochimistes qu'elle comprend que l'argan est une source d'opportunité d'ID. Dans le cas du chimiste, c'est le comportement d'observateur actif de ce dernier qui, dès le départ, le conduit à initier une mission d'exploration en France et, par la suite, à « jouer une carte »: exploiter une opportunité d'ID future.

Sur la base de ce comportement d'observateur actif, chaque dirigeant peut entrevoir une opportunité d'ID. Il peut concevoir un projet d'ID afin de résoudre le problème qui l'interpelle ou encore anticiper l'épidémie qui « n'existe » pas encore au Québec. Dès lors, les dirigeants doivent activer le rôle d'entrepreneur durable afin de saisir et de transformer de façon concrète les opportunités d'ID détectées en projets d'ID.

\subsection{L'entrepreneur durable}

L'analyse des données révèle la présence de deux entrepreneurs durables qui prennent la décision d'activer des projets d'ID à partir de la découverte (cas de la cosméticienne) ou de l'exploitation (cas du chimiste) d'une opportunité d'ID. Puisqu'au travers des informations recueillies et de l'interprétation qu'ils en font ainsi qu'au fil des interactions sociales, les dirigeants développent une connaissance des enjeux de la durabilité, ils sont bien placés pour percevoir les opportunités d'ID que ces enjeux comportent. Ainsi, c'est en agissant comme des entrepreneurs durables qu'ils sont capables de voir où se présentent de nouvelles opportunités, de détecter où se cachent les possibilités de nouveaux produits et services, et de créer des projets pour lancer ces produits et services.

Par exemple, en ce qui concerne la cosméticienne équitable, la démarche consistant à prendre le risque de racheter tout le stock d'huile d'argan et par la suite de se questionner sur ce qu'elle peut en faire, l'oblige à rencontrer en 2005, grâce à un de ses amis qui est aussi un écologiste, une firme de biochimistes. Cette rencontre permet à la dirigeante de découvrir que l'huile d'argan est en fait une opportunité à la fois scientifique et économique (pour les biochimistes) ainsi qu'écologique (pour la cosmétologue). Partant de là, elle prend la décision de mettre au point un projet d'entreprise en vue d'exploiter cette opportunité pour des raisons non seulement économiques (valoriser l'huile d'argan) mais également sociales (aider les femmes).

« J'ai racheté toute la production d'huile d'argan de ces femmes pour éviter qu'elles soient expulsées. J'ai ensuite rapporté ce stock d'huile au Québec. Mais en réalité, je ne savais pas quoi en faire. Pendant plusieurs mois, j'avais ces quantités de marchandise en réserve sous la main et je me questionnais sur ce que je pouvais en faire. Avec l'aide d'un ami déjà sensibilisé aux questions environnementales et sociales (qui deviendra vice-président de la compagnie), j'ai rencontré des biochimistes qui m'ont fait comprendre que cette matière première (l'huile d'argan) était une opportunité d'abord scientifique (pour eux) puis économique qui se présentait à moi : montrer aux québécoises les vertus de l'huile d'argan pour la peau à travers un cosmétique ».

Ainsi, les actions de la cosméticienne évoluent au fil de ses rencontres et se transforme graduellement en actions plus stratégiques : la cosméticienne doit montrer aux québécois les 
bienfaits de l'argan parce qu'elle pense que ces derniers peuvent en profiter à travers un cosmétique qu'elle pourrait développer et commercialiser.

Dans le cas du chimiste durable, grâce au concours d'anciens collègues, le dirigeant perçoit dans l'enjeu de la durabilité, soit environnemental (pollution de l'air) et social (santé publique), que représente la bactérie une opportunité qui puisse à la fois prévenir les risques d'épidémie au Québec en plus de constituer une technologie plus performante non seulement au plan économique mais également au plan strictement environnemental et sanitaire. Ici, le comportement du dirigeant se traduit par l'exploitation d'une opportunité future et qui débute par une redéfinition de la stratégie de l'entreprise et la constitution d'une équipe interne composée d'un directeur technique, d'une coordonnatrice en développement durable, d'un responsable des applications technologiques et de techniciens de laboratoires.

« J'ai ajouté le terme "durable" à mon titre de vice-président innovation de toutes les divisions de l'entreprise pour montrer que c'était une de mes préoccupations et la nouvelle orientation de l'entreprise. Certes, les crises passées concernant la bactérie m'ont inspiré car les tours de refroidissement sont très utilisées au Québec. Mais après, j'ai pensé avec une équipe composée de techniciens de laboratoire, d'un directeur technique, d'une coordonnatrice en développement durable et d'un responsable des applications technologiques qu'il serait opportun de concevoir une technologie de prévention et de traitement de cette bactérie bien qu'elle n'existe pas encore au Québec. Cette démarche nous permettra d'être à l'avant-garde dans notre secteur ».

Nous constatons en particulier que le rôle d'entrepreneur durable tel qu'activé par nos dirigeants de PME ressemble à celui d'un bricoleur. En effet, en cherchant à acquérir auprès de certains acteurs plus d'informations et de connaissances sur les enjeux de durabilité, et en saisissant encore une fois avec le soutien de ces acteurs les opportunités d'ID que comportent ces enjeux, puis en transformant ces opportunités en projets d'ID, les dirigeants de PME réorganisent les choses à leur disposition (p. ex.: l'huile d'argan, le titre de leur poste, l'orientation de leur entreprise) afin de créer de nouvelles opportunités de produits et services plus durables. À nouveau, leurs comportements ne sont pas uniquement le produit de traits mais aussi la résultante d'interactions contextualisées au sein desquelles ils « créent » concrètement et de manière spécifique leur rôle d'entrepreneur durable. Par la suite et du fait de certaines limites au niveau de leurs ressources et compétences, nos dirigeants de PME vont agir comme des leaders durables en créant des liens de collaborations avec des acteurs dont les ressources et compétences sont cruciales pour la mise en œuvre de leurs projets d'ID.

\subsection{Le leader durable}

Afin d'assurer la réalisation de leurs projets d'ID, les dirigeants de PME rencontrés vont au contact et mobilisent différents acteurs dont la collaboration s'avère nécessaire pour l'existence de l'ID (en raison des ressources et compétences dont ces derniers disposent). Ils vont aussi modifier les caractéristiques de leurs ID afin que celles-ci prennent en compte les intérêts, demandes ou préoccupations de ces acteurs. Cette démarche implique que les dirigeants aient la capacité de réunir ces personnes autour d'une vision commune de l'ID. Ainsi, ces dirigeants agissent comme des leaders durables parce qu'ils sont capables d'initier et d'entretenir des collaborations à l'intérieur comme à l'extérieur de leur organisation avec des 
gens sensibles à différents enjeux de la durabilité. Tout en facilitant la réalisation des ID, ces collaborations donnent une légitimité à celles-ci. Ni pour la cosméticienne et ni pour le chimiste, ce rôle est un script écrit à l'avance qui déterminerait leurs comportements. Le rôle de leader durable sera au moins en partie une création dont on trouve les fondements dans les interactions et le contexte au sein duquel elles ont cours.

Par exemple, dans le cas de la cosméticienne équitable, après avoir contacté les biochimistes, elle va mobiliser une cosmétologue et, plus tard, deux organisations de certification de produits équitables. En effet, insatisfaite en partie de la proposition de la firme de biochimistes qui consiste à combiner l'huile d'argan à des excipients synthétiques (dérivés du pétrole et par conséquent, dangereux pour la santé) habituellement utilisés dans le secteur du cosmétique, elle se rapproche d'une cosmétologue qui voit les choses de façon plus écologique. Cette dernière privilégie plutôt un cosmétique entièrement naturel et sécuritaire, soit fait essentiellement à base d'huile d'argan. Il faut préciser qu'un tel cosmétique, bien que non nocif pour la santé, a des propriétés (composés de grosses molécules) qui ne facilitent pas une bonne pénétration dans la peau. Or, dans le cas des cosmétiques, les consommateurs s'attendent à des produits qui ont des effets rapides, conviennent à différents types de peau et sont adaptés au climat. C'est pourquoi, pour la firme de biochimistes il faut développer un cosmétique qui combine l'huile d'argan à des excipients synthétiques afin de s'assurer d'un produit rentable économiquement qui peut également rivaliser avec la concurrence. Ceci étant dit, en plus d'être écologique et économiquement rentable, la dirigeante tient à un cosmétique équitable parce qu'elle se préoccupe du bien-être socioéconomique des femmes avec qui elle souhaite maintenir ses relations. Pour acquérir ce label équitable et prouver que l'huile est payée à un « juste prix » (un prix qui garantisse un revenu qui reflète la réalité des femmes productrices d'huile d'argan), elle implique des organisations de certification de produits équitables. Ici, il faut noter que dans toutes ces démarches de collaboration l'objectif de la dirigeante est de bénéficier des ressources et compétences de tous ces acteurs et par conséquent de favoriser une ID dont l'existence sera le fruit des efforts conjoints de chaque acteur mobilisé. Cet objectif de la dirigeante se reflète dans le cosmétique à la fois écologique et équitable qu'elle finit par développer avec le soutien de ces acteurs y compris celui des femmes productrices d'huile d'argan.

Dans le cas du chimiste durable, les enjeux environnementaux (pollution de l'air) et sociaux (santé publique) que représente la bactérie ne sont pas encore d'actualité au Québec alors qu'il observe leur existence lors d'un voyage en France en 2010 et décide, suite aux échanges avec ses hôtes, de profiter de cette opportunité de développer une ID. Par conséquent, il faut développer une ID dont la légitimité devra être renforcée par une législation tout comme en Europe. Ainsi en 2011, le directeur technique, la coordonnatrice en développement durable et le responsable des applications et technologies de l'entreprise obtiennent, en mobilisant leur association professionnelle ${ }^{2}$, une rencontre avec les députés.

" Notre argumentaire est le suivant : Il n'existe aucune règlementation en vigueur au Québec pour assurer la surveillance d'une bactérie meurtrière dans les installations des bâtiments. Une législation pourra amener les entreprises à recourir à des analyses périodiques et préventives afin de réduire les risques de contamination dans la population

\footnotetext{
${ }^{2} \mathrm{Au}$ Québec, une rencontre n'est possible avec les députés que si l'entreprise figure au Registre des lobbyistes sous l'égide d'une association.
} 
et de pollution de l'environnement. Mais, pour plusieurs députés, il ne faut pas effrayer la population avec une législation. Et de toutes les façons il existe une solution pour combattre la bactérie si elle survenait. Mais la solution dont parlent les députés utilise une technologie d'analyse standard qui a montré ses limites même en France étant donné le délai de réponse très long et de l'impact environnemental négatif des traitements ».

Ainsi, le projet d'ID de l'entreprise sera mis en berne jusqu'à ce que survienne, en 2012, une crise au Québec impliquant la bactérie à l'origine des tragiques évènements qu'il avait observés en France et en Europe. Comme en Europe, cette crise fait une dizaine de morts en plus d'affecter une centaine de personnes. En 2013, la Régie du bâtiment décide d'élaborer un projet de règlement en s'appuyant sur les recommandations de l'association professionnelle à laquelle appartient la PME. En fait, l'association préconise que la solution pour combattre la bactérie comporte une méthode de détection rapide et de traitement non toxique, implicitement, comme celle que la PME a mise au point. Les recommandations de l'association donnent alors l'occasion à la PME de " réveiller » et d'adapter son projet d'ID pour convenir au projet de règlement de la Régie du bâtiment mais également aux procédures des services de santé publique.

En somme, comme l'illustrent les deux cas étudiés, l'activation du rôle de leader durable vise à se donner des ressources et compétences pour réaliser l'ID. Cette démarche débute par la mobilisation d'acteurs-clés, mais surtout par la construction avec ces derniers d'un dénominateur commun tout en veillant à ce qu'il soit suffisamment significatif pour que les acteurs gardent un intérêt pour le projet. Ainsi, les caractéristiques de l'ID sont la version matérielle du réseau social et technique mis en place pour la faire exister. La mission en France, la définition d'une nouvelle orientation vers la durabilité, l'initiative de rencontrer les députés, les négociations avec les organismes de financement sont quelques actions du leader durable qui ont permis à l'ID d'exister. De même, dans le cas de la cosméticienne, c'est son action consistant à prendre le risque d'acheter tout le stock d'huile d'argan, à rencontrer des biochimistes, une cosmétologue et des organisations de certification, qui a déterminé l'existence de l'ID. Ainsi, pour que toutes ces collaborations fonctionnent, les dirigeants de PME ont adopté un comportement que nous qualifierons de réseauteur parce qu'il consiste à créer et maintenir un ensemble de relations hétérogènes entre des acteurs-clés aux intérêts environnementaux, sociaux et économiques divers (et dont les ressources et compétences sont cruciales pour le développement de l'ID) autour d'une vision co-construite de l'ID.

Pour créer ces relations hétérogènes, les dirigeants doivent faire des ajustements qui entrainent une recombinaison des caractéristiques de leurs ID. Par exemple, dans le cas de la cosméticienne, elle propose un cosmétique à la fois écologique (mobilisation d'une cosmétologue) et équitable (préoccupation pour le bien-être socioéconomique des femmes ; recours à des organisations de certifications) ayant un potentiel économique (implication de la firme de biochimistes). Dans le cas du chimiste, le dirigeant doit non seulement adapter son discours pour attirer l'attention des députés, mais également ajuster sa solution pour convenir au projet de règlement de la Régie du bâtiment et aux procédures des services de santé publique. Ainsi, en agissant comme des réseauteurs, nos dirigeants construisent un noyau dur, un réseau d'acteurs-clés autour de leurs ID. Ici, il est difficile de comprendre ce que font les dirigeants en prenant uniquement en considération leurs traits et en ramenant leurs actions à 
une manifestation de ceux-ci. Pour les comprendre, il faut également prendre en compte les contextes spécifiques dans lesquels les traits s'expriment dans des comportements.

En définitive, en plus de démontrer l'existence des trois rôles, les résultats de notre étude montrent que pour réaliser leurs ID nos dirigeants de PME doivent activer trois comportements spécifiques comme l'illustre la figure 1: observateur actif, bricoleur, réseauteur. Les rôles n'existent pas indépendamment de ces comportements. Ils sont au moins en partie " créés » par ces derniers ; ils en émergent (Mintzberg, 1984; O'Gorman et al., 2005).

\section{Figure 1: Modèle intégré des rôles du dirigeant de PME dans le développement des ID}

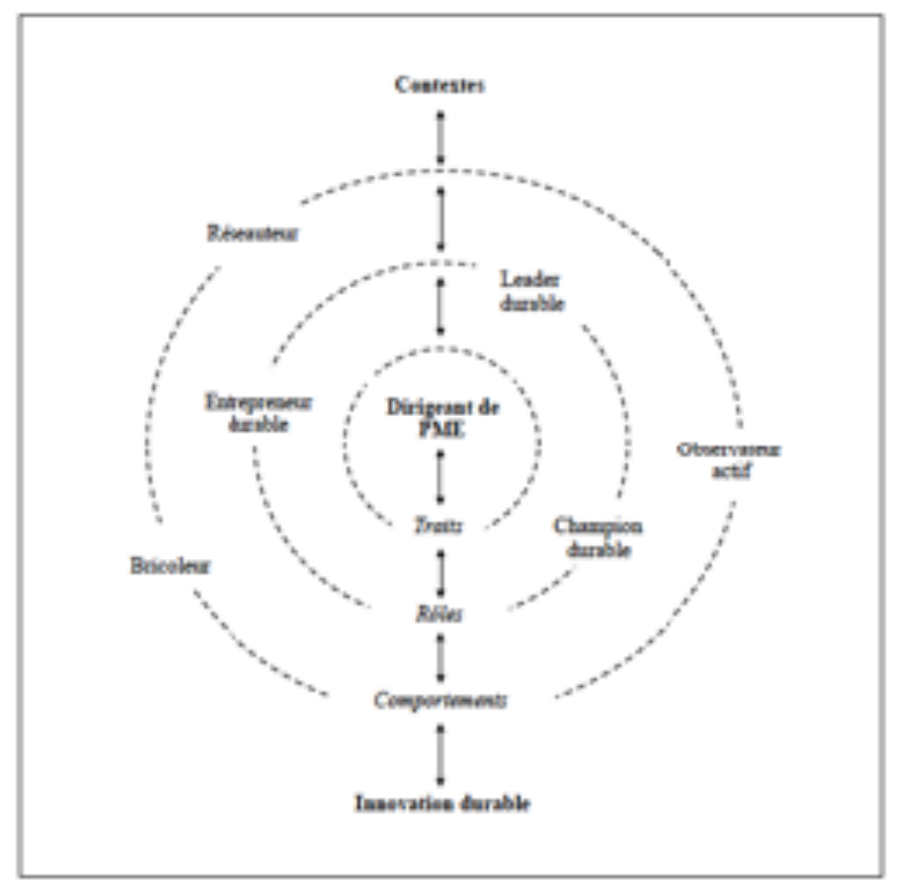

De plus, nos dirigeants doivent démontrer leur capacité à passer d'un comportement à un autre. Cette dynamique comportementale traduit bien les changements de rôles des dirigeants. C'est également à travers cette dynamique comportementale que les rôles de champion durable, d'entrepreneur durable et de leader durable sont interreliés. Et, comme nous l'avons $\mathrm{vu}$, ils prennent forme en étroite relation avec les contextes dans lesquels ils se déploient. Autrement dit, le tout n'est pas linéaire mais superposé.

\section{Discussions et conclusion}

La question spécifique de cette étude était de comprendre comment le dirigeant de PME concilie dans des comportements spécifiques les rôles de champion durable, d'entrepreneur durable et de leader durable afin de développer une ID. À la suite de l'analyse des données et des résultats de notre étude, nous sommes à même d'identifier des comportements spécifiques 
tenus par nos dirigeants de PME selon les contextes et de montrer comment ceux-ci sont activés et conciliés durant le développement des ID. Nous proposons les réponses suivantes :

- Dans le rôle de champion durable, c'est le comportement d'observateur actif qui prédomine. Nos dirigeants perçoivent dans leur environnement une information liée à un enjeu de durabilité prioritaire pour eux. Mais le comportement d'observateur actif ne s'observe pas exclusivement alors que le dirigeant assume son rôle de champion durable. Il peut également être présent lorsque celui-ci joue son rôle d'entrepreneur durable et que, par exemple, il recueille de l'information qui, dans le cadre d'interactions contextualisées, lui sert à construire une opportunité et un projet d'ID à partir de l'enjeu identifié.

- Dans le rôle d'entrepreneur durable, c'est le comportement de bricoleur qui prédomine. Dans leurs interactions avec différents acteurs et avec différents objets physiques et cognitifs, ils reconnaissent et saisissent une opportunité d'ID puis bricolent un projet d'ID. Le dirigeant rassemble et organise des morceaux disparates pour se faire une idée du tout (Millier, 2016), soit de son ID et du projet qui la fera exister. Une des dimensions essentielles de ce bricolage est de type relationnel puisque nos dirigeants doivent se doter de ressources et de compétences pour réaliser leurs ID en activant le rôle de leader durable.

- Dans le rôle de leader durable, c'est le comportement de réseauteur qui prédomine. Ainsi en mobilisant et en intéressant des acteurs externes au projet d'ID, nos dirigeants créent et maintiennent un réseau restreint d'acteurs-clés qui donneront à l'ID ses caractéristiques définitives. Pour créer le réseau, les dirigeants doivent réconcilier les différents intérêts en faisant de nouvelles combinaisons ou en adaptant leurs ID. Ce faisant, le réseauteur cherche à légitimer l'ID afin de stabiliser ses caractéristiques et de rendre irréversible sa réalisation.

Partant de là, nos résultats montrent 1) que les rôles de champion durable, d'entrepreneur durable et de leader durable sont essentiels au dirigeant de PME qui s'engage dans le développement des ID, 2) et que ces rôles prennent forme à travers trois comportements spécifiques: observateur actif, bricoleur et réseauteur.

Premièrement, la présence des trois rôles est supportée par les recherches qui le plus souvent présentent un seul rôle, privilégient une approche fonctionnaliste de celui-ci (inspirée, par exemple de Fayol) et omettent d'en présenter une vision intégrée. À ce propos, nos résultats montrent que c'est la mise en œuvre de chacun des trois rôles par le dirigeant de PME selon les situations qui se présentent qui favorise la réalisation des ID : ces rôles sont donc intégrés. Par exemple, l'activation du rôle de champion durable devient plus importante au moment de la recherche, de l'identification et de l'interprétation des informations liées aux enjeux de la durabilité. Mais ce rôle tout comme chacun des deux autres n'est pas exclusif à une situation: ils deviennent davantage pertinents que d'autres selon les situations. Par conséquent, une analyse intégrée de ces rôles permet de mieux comprendre leurs interactions et leur relation complémentaire avec ce qui constitue le résultat des actions du dirigeant de PME (Verstraete et Saporta, 2006), soit le projet d'ID puis finalement l'ID. Dans ce sens, ces résultats rejoignent ceux de Bossink (2013), mais s'en éloignent dans la mesure où les travaux de ce dernier portent sur des grandes entreprises et les rôles y sont joués par différents individus. Nos résultats indiquent plutôt qu'en contexte de PME ces rôles sont tous exercés par le 
dirigeant parce que son entreprise et lui ne font souvent qu'un (Julien et Marchesnay, 1996) et parce qu'en raison de la petite taille de ce type d'organisation et des façons de faire, la prise de décision et les actions en matière d'innovation lui reviennent directement (Rothwell et Dodgson, 1991). Plus généralement, le constat selon lequel le dirigeant de PME joue à la fois les rôles qu'il perçoit comme étant cruciaux pour son entreprise est également soutenu par les travaux d'O'Gorman et al. (2005). Par ailleurs, nos résultats permettent de voir que sur la base d'une approche comportementale (Bird et al., 2012) et émergente (Carlson, 1951; Mintzberg, 1984) du rôle, les dirigeants de PME de notre étude sont capables de passer d'un rôle à un autre parce que les comportements grâce auxquels ils sont activés s'expriment 1) dans des interactions avec un réseau d'acteurs qui est lui-même lié à tous les aspects de l'ID et 2) dans un contexte spécifique où les évènements ont cours indépendamment de la volonté du dirigeant et des autres acteurs. Ce faisant, le rôle ne peut plus être vu comme un script à réciter découlant des traits du dirigeant. Ce dernier a une certaine flexibilité dans la manière dont il définit le rôle et le met en œuvre. Cette dynamique du comportement de nos dirigeants traduit la dimension interactionniste du concept de rôle (Biddle, 1986; Fondas et Stewart, 1994) puisque le rôle prend forme à travers des interactions contextualisées.

Deuxièmement, nos résultats permettent d'identifier l'observateur actif, le bricoleur et le réseauteur comme trois comportements spécifiques qui montrent que, dans le développement d'une ID, le dirigeant de PME fait ceci parce qu'il se comporte comme cela. Ces comportements ont été nommés ainsi en raison de leur équivalence avec des concepts existants dans la littérature en science de la gestion. D'abord, selon Mintzberg (1984), l'observateur actif est « [...] en permanence en train de chercher et recevoir des informations qui lui permettent de mieux comprendre ce qui se passe dans son organisation et son environnement. [...] Il utilise un certain nombre de moyens pour acquérir une meilleure compréhension des tendances de son environnement et pour s'informer des idées nouvelles »(pp. 78-79). Ces actions décrivent bien le rôle de champion durable dans notre étude. Ensuite, le bricoleur est celui qui fait du bricolage défini par Baker et Nelson (2005) comme " making do by applying combinations of resources at hand to new problems and opportunities » (p. 33). Le comportement de bricoleur caractérise tant les actions de l'entrepreneur que la manière avec laquelle il développe des innovations (Fisher, 2012). Jaouen et Nakara (2014) utilisent le terme de bricoleur pour comprendre les pratiques des dirigeants de microfirmes (moins de cinq employés) en matière de système d'information. Pour ces auteurs, le bricoleur est celui qui fait preuve d'écoute, combine les ressources qu'il a en mains, crée ou exploitent de nouvelles opportunités, utilise les idées des différentes parties prenantes, recherche leur feedback et procède à d'éventuelles corrections (Jaouen et Nakara, 2014). C'est ce qu'ont fait les dirigeants de PME de notre étude. Par exemple, la cosméticienne écoute les femmes et rachète l'huile d'argan, utilise les idées des biochimistes et de la cosmétologue, procède à des ajustements suite à leur feedback. Le chimiste utilise les idées de ses partenaires français, exploite au Québec l'opportunité que représente la bactérie au Québec, effectue des modifications sur la solution préventive afin de faciliter son acceptation par les députés, les services de santé publique et la Régie du bâtiment. Par conséquent, nous pouvons qualifier les dirigeants de notre étude de bricoleurs. Enfin, le réseauteur est celui qui acquière et échange des ressources à travers un réseau d'acteurs externes qu'il mobilise (Mintzberg, 1984) dans le but de réaliser l'innovation. Ici, il faut noter que comparativement au bricoleur qui se "débrouille » avec ce qu'il a en mains, nos 
dirigeants de PME vont, en tant que réseauteur, chercher ailleurs ce qu'ils ne possèdent pas afin de poursuivre le bricolage entamé dans le rôle d'entrepreneur durable. Le réseau construit par le dirigeant comprend souvent des acteurs hétérogènes et dont les intérêts peuvent être différents voire divergents. Dans ces conditions et comme le souligne Akrich, Callon et Latour (1988), il revient au réseauteur de choisir et d' "intéresser » les acteurs dont les ressources sont cruciales pour le succès de l'innovation, puis de construire avec eux un compromis social (convergence des intérêts) et technique (adaptation des caractéristiques de l'innovation) satisfaisant. C'est ce qu'ont fait nos dirigeants de PME dans leur rôle de leader durable et c'est pour ces actions que nous les qualifions de réseauteur.

Partant de ce qui précède, notre principale contribution théorique tient au fait que lorsque l'attention est portée sur les comportements, la dynamique et la complémentarité entre les trois rôles sont telles que ceux-ci pourraient être considérés comme les trois dimensions d'un même construit, soit l'éco-innovateur. Cet enchâssement des trois rôles au sein d'un même construit a l'avantage d'illustrer leur interdépendance étroite et de rendre les frontières entre eux plus fluides. Chacun des rôles attire davantage l'attention sur un des comportements selon les situations. Mais ces comportements ne sont pas isolés les uns des autres. Par exemple, 1) bien que le comportement de réseauteur soit davantage lié au rôle de leader durable qui consiste à créer des liens de collaborations, il participe également 2) au rôle d'entrepreneur durable qui, à travers le comportement de bricoleur, va " construire » des opportunités et un projet d'ID avec des acteurs présents dans un réseau partagé, 3) ainsi qu'au rôle de champion durable qui peut à travers ce réseau acquérir des informations liées à des enjeux de la durabilité en s'appuyant sur le comportement d'un observateur actif. Cette contribution fait appel à des recherches futures sur le construit d'éco-innovateur qui caractérise en contexte de PME l'ensemble des rôles des dirigeants engagés dans le développement des ID. Nous suggérons plus particulièrement que des recherches s'intéressent au contexte des PME de plus grande taille ou issues d'autres secteurs (p. ex.: high-tech) afin de voir s'il y a des différences dans l'approche de l'éco-innovateur. Il serait également utile de pousser plus loin le concept de bricolage chez l'éco-innovateur en s'intéressant aux formes qu'il dans le contexte des PME et des ID. La théorie du bricolage de Levi-Strauss (1966) peut être ainsi revisitée afin de mieux comprendre certaines pratiques d'ID adoptées par des entrepreneurs ou des dirigeants de PME.

En termes d'implications managériales, les dirigeants de PME qui souhaitent développer des ID doivent posséder et mettre en œuvre un ensemble de comportements qui, dans le contexte des ID et des PME de notre étude, se sont avérés bénéfiques à la fois pour " démarrer » le processus d'ID et pour le fluidifier. Les entrepreneurs et dirigeants de PME peuvent adopter et renforcer leurs comportements dans l'un ou l'autre des trois principaux rôles ou identifier, parmi leurs collaborateurs internes, ceux qui vont mieux les traduire lorsqu'ils s'engagent dans une démarche d'ID. L'objectif est d'amplifier les chances de succès de leurs ID en confiant « les bons rôles aux bonnes personnes » et en adoptant « les bons comportements au bon moment ». Dans ce sens, les organisations intervenant en appui à l'innovation peuvent introduire dans leur accompagnement des entrepreneurs et dirigeants des façons de faire et des activités visant à faciliter l'émergence des comportements associés aux trois rôles. Par ailleurs, l'illustration que nous avons faite à travers l'étude de deux cas de PME peut être utilisée par ces derniers pour donner une indication de l'utilité de notre modèle intégré et pour 
donner une vision systémique de ce que des dirigeants de PME s'engageant dans le développement des ID doivent s'attendre à faire.

Pour finir, il faut signaler quelques limites de notre recherche. Parmi celles-ci, l'utilisation de deux cas représentatifs ou typiques (Yin, 2003) répondait à des critères de validité interne. Compte tenu de la validité externe limitée de notre étude, la réplication de celle-ci dans des contextes variés, dans des pays différents, dans plusieurs cas d'ID et de PME serait souhaitable. Par ailleurs, malgré l'éclairage que nous avons apporté sur les rôles du dirigeant dans le développement des ID, nous n'avons pas été en mesure de comparer ce processus avec celui qui prévaut dans un contexte d'innovation plus classique afin de voir s'il y a des différences fondamentales. En d'autres termes, une comparaison des processus d'ID et d'innovation plus « classique » permettrait de faire ressortir davantage les particularités des ID et des rôles du dirigeant de PME.

\section{Références}

Adams, R., Jeanrenaud, S., Bessant, J., Denyer, D. et Overt, P. (2016). Sustainabilityoriented Innovation: A Systematic Review. International Journal of Management Reviews, $18,180-205$.

AKrich, M., CAllon, M. et LATOuR, B. (1988). À quoi tient le succès des innovations. Partie 1 : L'art de l'intéressement. Gérer et Comprendre, 21.

AUTEUR, (2015).

Auteurs, (2010).

BAKER, T., et NELSON, R. E. (2005). Creating something from nothing: Resource construction through entrepreneurial bricolage. Administrative Science Quarterly, 50 (3), 329-66.

ANDERSON, L. M. et BATEMAN, T. S. (2000). Individual Environmental Initiative: Championing Natural Environmental Issues in U.S. Business Organizations. Academy of management journal, 43(4), 548-570.

BANDURA, A. (2012). On the functional properties of perceived self-efficacy revisited. Journal of Management, 38, 9-44.

BANERJEE, S. (2001). Managerial perceptions of corporate envrionmentalism: interpretations from industry and strategic implications for organizations. Joumai of Management Studies, $38(4), 489-514$.

BANSAL, P. et Song, H.-C. (2017). Similar but not the same: differ entiating corporate sustainbinility from corporate responsibility. Academy of Management Annals, 11(1) 105149.

BERCHICCI, L. (2005). The green entrepreneur's challenge. The influence of environmental ambition in new product development. Thèse de doctorat, Delft University of Technology, Delf. 
BIDDLE, B. J. (1986). Recent Development in Role Theory. Annual Review of Sociology, 12, $67-92$.

BIRD, B., SCHJOEDT, L. et BAUM, J. (2012). Editor's Introduction. Entrepreneurs' Behavior: Elucidation and Measurement. Entrepreneurship: Theory \& Practice, 36(5), 889-913

Bos-Brouwers, H. E. J. (2009). Corporate Sustainability and Innovation in SMEs: Evidence of Themes and Activities in Practice. Business Strategy and the Environment, 1-20.

Bossink, B. A. (2013). Eco-innovation and sustainability management. Routledge: New York and London.

BRÍO, J. A. D. et JÙNQUERA, B. (2003). A review of the literature on environmental innovation management in SMEs: implications for public policies. Technovation, 23(12), 939-948.

CARlson, S. (1951). Executive Behaviour: A Study of the Work Load and the Working Methods of Managing Directors. Stockholm: Stromberg

DEAN, T. et MCMulLen, J. (2007). Toward a theory of sustainable entrepreneurship: reducing environmental degradation through entrepreneurial action. Journal of Business Venturing, $22(1), 50-76$.

Desmarais, C. et ABORD DE Chatillon, E. (2010). Le rôle de traduction du manager : Entre allégeance et résistance. Revue française de gestion, 6(205), 71-88.

Egri, C. P. et Frost, P. J. (1994). Leadership for environmental and social change. Leadership quartely, 5(3-4), 195-200.

Egri, C. P. et Herman, S. (2000). Leadership in the north american environmental sector: values, leadership styles, and contexts of environmental leaders and their organizations. Academy of management journal, 43(4), 571-604.

FISHER, G. (2012). Effectuation, Causation, and Bricolage: A Behavioral Comparison of Emerging Theories in Entrepreneurship Research. Entrepreneurship Theory and Practice, 36, 1019-1051

FONDAS, N. et STEWART, R. (1989). Study of managerial jobs and behaviour: The ways forward. The Journal of management studies, 26(1), 1-10.

FUSSLER, C. et JAMES, P. (1996). Eco-Innovation: a Break thorough Discipline for Innovation and Sustainability. London: Pitman.

Gadenne, D. L., Kennedy, J. et McKeiver, C. (2009). An Empirical Study of Environmental Awareness and Practices in SMEs. Journal of Business Ethics, 84, 45-63.

GARTNER, W. B. (1989). Who is entrepreneur? is the wrong question. American Journal of Small Business, 12, 11-32.

GAUTHIER, B. et COLlaBORATEURS (2006). Recherche sociale, de la problématique à la collecte des données (3e éd.). QC, Canada : Presses de l'université du Québec 
GIRIN, J. (1990). Analyse empirique des situations de gestion : éléments de théorie et de méthode. Dans Martinet A.C. (coord.) Épistémologie et Sciences de Gestion (p. 141-182). Economica: Paris.

Greenhaus, J. H. et Powell, G. N. (2006). When work and family are allies : A theory of work-family enrichment. Academy of Management Review, 31(1), 72-92.

HALL, J. (2002). Sustainable development innovation; a research agenda for the next 10 years. Editorial for the 10th Anniversary of the Journal of Cleaner Production, Journal of Cleaner Production, 10(3), 195-196.

Hall, J et VRedenburg, H. (2003). The Challenges of Innovating for Sustainable Development. MIT Sloan Management Review, 45(1), 61-68.

Hansen, O., Sondergard, B. et Meredith, S. (2002). Environmental Innovations in Small and Medium Sized Enterprises. Technology Analysis \& Strategic Management, 14(1), 37-56.

JAOUEN, A. et NAKARA, W. A. (2014). Les systèmes d'information en microfirme: une approche par le bricolage organisationnel. Revue internationale PME, 27(3-4), 225-260.

JENKINS, H. (2009). A 'business opportunity' model of corporate social responsibility for small- and medium-sized enterprises. Business Ethics: A European Review, 18(1), 21-36.

Julien, P. A. et MARCheSnay, M. (1996). L'entrepreneuriat. Paris: Economica.

Klewitz, J. et HANSEN, E. G. (2014). Sustainability-Oriented Innovation of SMEs: A Systematic Review. Journal of Cleaner Production, 65, 57-75.

LARSON, A. L. (2000). Sustainable Innovation Through an entrepreneurship Lens. Business Strategy and the Environment, 9, 304-317.

Levi-Strauss, C. (1966). The savage mind. University of Chicago Press : Chicago.

Miles, M. B. et Huberman, M. A. (2003). Analyse des données qualitatives (2éd.). Paris: De Boeck.

Millier, P. (2016). L'ingénieur, le bricoleur et l'innovateur. Entreprendre \& Innover, 1(28), 48-62.

MinTZBERG, H. (1984). Le manager au quotidien : Les dix rôles du cadre. Paris: Les éditions d'organisation.

Moore, S. B. et MAnRing, S. L. (2009). Strategy development in small and medium sized enterprises for sustainability and increased value creation. Journal of Cleaner Production, 17, $276-282$

OCDE (2010). L'éco-innovation dans l'industrie : Favoriser la croissance verte, OECD Innovation Strategy. OECD Publishing.

O'Gorman, C. Bourke, S. et Murray, J. A. (2005). The nature of managerial work in small growth-orientated businesses. Small Business Economics, 25, 1-16. 
PARKER, C. M., REDMOND, J. et SimPSON, M. (2009). A review of interventions to encourage SMEs to make environmental improvements. Environment and planning C: Government and Policy, 27, 279-301. .

Patzelt, H. et ShePherd, D. A. (2010). Recognizing Opportunities for Sustainable Development. Entrepreneurship Theory and Pratice. 1-22.

Porter, M. E. et Kramer, M. (2006). Strategy and society. Harvard Business Review, 78-92.

RÉSEAU ENTREPRISE ET DÉVELOPPEMENT DURABLE (REDD) (2012). Canadian business sustainability challenges 2012. Rapport. Disponible sur http://nbs.net/wp-content/uploads/ Business-Sustainability-Challenges-2012.pdf

Rothwell, R. et Dodgson, M. (1991). External linkages and innovation in small and medium-sized enterprises. R\&D Management, 21(2), 125-138.

SAnDSTRÖM, G. et TINGSTRÖM, J. (2008). Management of radical innovation and environmental challenges. European Journal of Innovation Management, 11(2), 182-198.

SARKis, J., Cordeiro, J. J. et Brust, D. A. V. (2010). Facilitating Sustainable Innovation through Collaboration. Dans Joseph Sarkis (Eds.), Facilitating Sustainable Innovation through Collaboration: A Multi-Stakeholder Perspective (pp. 1-16). Springer Science.

Schaltegger, S. et Wagner, M. (2010). Sustainable Entrepreneurship and Sustainability Innovation: Categories and Interactions. Business Strategy and the Environment, 20(4), 222-237.

SCHAPER, M. (2002). Small firms and environmental management: Predictors of green purchasing in western australien pharmacies. International Small Business Journal. 20(3), 235-251.

SHARMA, S. (2000). Managerial interpretations and organizational context as predictors of corporate choice of environmental strategy. Academy of Management Journal, 43, 681-697.

SHRIVASTAVA, P. (1994). « Ecocentric leadership in the 21st century x. Leadership Quarterly, 5(3-4), 223-226.

SteVEnSON, H. et JARILlo, J. (1990). A paradigm of entrepreneurship: Entrepreneurial management. Strategic Management Journal, 11, 17-27.

THIÉTART, R. A. (2007) (dir.), Méthodes de recherche en management (2e. édition), Paris, Dunod.

TidD, J., Bessant, J. et PaViTt, K. (2006), Management de l'innovation: Intégration du changement technologique, commercial et organisationnel, Bruxelles, De Boeck Supérieur.

Triguero-Cano, A., Moreno-MondéJar, L, et Davia, M. A. (2013). Drivers of different types of eco-innovation in European SMEs. Ecological Economics, 92, 25-33. 
Van de Ven, A. H., Angle, H. L. et Poole, M. S. (2000). Research on the Management of Innovation: The Minnesota Studies. New York: Ballinger/Harper \& Row.

VERSTRAETE T. et SAPORTA, B. (2006). Création d'entreprise et entrepreneuriat. Editions de l'ARDEG, $518 \mathrm{p}$.

VIVES, A. (2006). Social and Environmental Responsibility in Small and Medium Enterprises in Latin America. The Journal of Corporate Citizenship, 21, 39-50.

Walker, B., Redmond, J., Sheridan, L., Wang, C. et Goeft, U. (2008). Small and Medium Enterprises and The Environment: Barriers, Drivers, Innovation and Best Practice: A Review of the Literature. Working Paper.

WATSON, J. (2007). Modeling the relationship between networking and firm performance. Journal of Business Venturing, 22(6), 852-874.

WrZeSniEwSKi, A. et DutTon, J. E. (2001). Crafting a job: Revisioning employees as active crafters of their work. Academy of Management Review, 26, 179-201.

YIN, R. K. (2003). Case Study Research: Design and Method (3rd ed.). Thousand Oaks, California: Sage Publications.

York, J. G. et VenKataraman, S. (2010). The entrepreneur-environment nexus : uncertainty, innovation and allocation. Journal of Business Venturing, 25, 449 - 463.

YUKL, G. (2001). Leadership in organizations. Englewood Cliffs, NJ: Prentice-Hall. 